\title{
A Reduced Basis Enrichment for the eXtended Finite Element Method
}

\author{
E. Chahine ${ }^{a}$, P. Laborde ${ }^{b}$ and Y. Renard $^{c 1}$ \\ ${ }^{a}$ Institut de Mathématiques, UMR CNRS 5215, GMM INSA Toulouse, Complexe scientifique \\ de Rangueil, 31077 Toulouse Cedex 4, France \\ ${ }^{b}$ Institut de Mathématiques, UMR CNRS 5215, Université Paul Sabatier, 118 route de Narbonne, \\ 31062 Toulouse Cedex 4, France \\ ${ }^{c}$ Université de Lyon, CNRS, \\ INSA-Lyon, ICJ UMR5208, LaMCoS UMR5259, F-69621, Villeurbanne, France
}

\begin{abstract}
This paper is devoted to the introduction of a new variant of the extended finite element method (Xfem) for the approximation of elastostatic fracture problems. This variant consists in a reduced basis strategy for the definition of the crack tip enrichment. It is particularly adapted when the asymptotic crack-tip displacement is complex or even unknown. We give a mathematical result of quasi-optimal a priori error estimate and some computational tests including a comparison with some other strategies.
\end{abstract}

Key words: fracture, finite element method, Xfem, reduced basis, error estimates.

AMS subject classification: 74R99, 74S05, 65N30.

\section{Introduction}

The aim of this paper is to introduce a new variant of the eXtended Finite Element Method (Xfem). This latter method was introduced in $[22,21]$ in order to bypass some difficulties coming from classical finite element strategies to approximate the deformation of cracked elastic bodies (refinement of the mesh around the crack tip, remeshing after crack propagation). In fact, many approaches have been developed in the past decades to try make the finite element methods more flexible for

\footnotetext{
${ }^{1}$ Corresponding author. E-mail: Yves.Renard@insa-lyon.fr
} 
such applications. The pioneer work is the one of Strang and Fix, 1973 [25] in which a nonsmooth enrichment of the finite element method is introduced using a cut-off function to limit the influence zone of the enrichment. Since then, different approaches had been analyzed such the Pufem (the Partition of Unity Finite Element Method) [20], the Arlequin method [9], the Gfem (Generalized Finite Element Method) [26], the Xfem and the patches enrichment approach [12].

The Xfem becomes popular due to its flexibility. It consists in the enrichment of the classical finite element basis by a step function along the crack line, to take into consideration the discontinuity of the displacement field, and by some nonsmooth functions to represent the asymptotic displacement in a vicinity of the crack tip. This enrichment strategy allows the use of a mesh independent of the crack geometry. Since the introduction of the Xfem, a rapidly growing literature have been produced in order to explore or improve the method (see [16] [2] and the references therein for instance).

So far, modeling cracked domains with Xfem approaches required a knowledge on the exact nonsmooth displacement at the crack tip. This becomes a serious difficulty when the asymptotic displacement at the crack tip is complicated or even unknown, e.g. for a bi-material interface crack. These issues were already pointed out in [6] where we introduced the Spider Xfem that needs only a partial knowledge on the asymptotic displacement.

Furthermore, the so-called Reduced Basis method has been introduced by Noor and Peters [23] in 1980. Then, the idea was developed by Maday and Rønquist in [19]. This method uses some pre-computed generic solutions as basis functions for the approximation. These numerical functions are obtained, once for all, for some values of parameters depending on the material characteristics and geometric properties. Meanwhile, a sufficient number of pre-computed functions are necessary. These pre-computed functions have also to be sufficiently independent the ones from the others. A good approximation of the solution can be obtained with this method with only a few number of pre-computed functions.

Inspired by the Reduced Basis method, we introduce in this paper, what we call the Reduced Basis enrichment eXtended Finite Element Method (or RB-Xfem) which is an Xfem variant with a reduced basis type nonsmooth enrichment. The main objective is to be able to treat unknown crack tip asymptotic displacement. In this method, the exact nonsmooth enrichment is replaced by some pre-computed finite element functions on very refined meshes. These functions should be able to approximate accurately the asymptotic displacement. They are computed once for all and are rather few in comparison with the classical Xfem enrichment in complex situations.

The outline of the paper is the following. Section 2 describes the problem and the new variant RB-Xfem. In section 3, a quasi-optimal result of convergence is obtained. Finally, in section 4, some numerical experiments are presented for an homogeneous isotropic crack and for a bimaterial interface crack. 


\section{Reduced extended finite element method via a cut-off func- tion}

Let $\Omega \subset \mathbb{R}^{2}$ be a bounded cracked polygonal domain which represents the reference configuration of a cracked linearly isotropic elastic body in plane stress approximation. The boundary of $\Omega$, denoted $\partial \Omega$, is partitioned into three parts $\Gamma_{D}, \Gamma_{N}$ and $\Gamma_{C}$ open in $\Omega$. A Dirichlet condition is prescribed on $\Gamma_{D}$ and a Neumann one on $\Gamma_{N}$ and $\Gamma_{C}$. The part $\Gamma_{C}$ of the boundary is representing the crack (see Figure 1). We denote $x^{*}$ the crack tip and $\Omega^{*}=\Omega \cup \Gamma_{C} \cup\left\{x^{*}\right\}$ the uncracked domain.

Let

$$
\mathbf{V}=\left\{\mathbf{v} \in H^{1}\left(\Omega ; \mathbb{R}^{2}\right) ; \mathbf{v}=0 \text { on } \Gamma_{D}\right\}
$$

be the space of admissible displacements and let us define

$$
\begin{gathered}
a(\mathbf{u}, \mathbf{v})=\int_{\Omega} \sigma(\mathbf{u}): \varepsilon(\mathbf{v}) d x, \quad l(\mathbf{v})=\int_{\Omega} \boldsymbol{\xi} \cdot \mathbf{v} d x+\int_{\Gamma_{N}} \boldsymbol{\zeta} . \mathbf{v} d \Gamma, \\
\sigma(\mathbf{u})=\lambda \operatorname{tr} \varepsilon(\mathbf{u}) I+2 \mu \varepsilon(\mathbf{u}),
\end{gathered}
$$

where $\sigma(\mathbf{u})$ is the stress tensor, $\varepsilon(\mathbf{u})$ is the linearized strain tensor, $\boldsymbol{\xi}$ and $\boldsymbol{\zeta}$ denote some given force densities on $\Omega$ and $\Gamma_{N}$ respectively, finally, $\lambda>0, \mu>0$ are the Lamé coefficients (which may have different values on one side and on the other side of the crack for the bi-material case). The elastostatic problem reads as

$$
\text { Find } \mathbf{u} \in \mathbf{V} \text { such that } a(\mathbf{u}, \mathbf{v})=l(\mathbf{v}) \quad \forall \mathbf{v} \in \mathbf{V} .
$$

We suppose that the solution $\mathbf{u}$ to this problem is a sum of a regular part and a nonsmooth part

$$
\mathbf{u}=\overline{\mathbf{u}}_{r}+\mathbf{u}_{s}
$$

such that $\overline{\mathbf{u}}_{r}$ is regular in the sense

$$
\overline{\mathbf{u}}_{r} \in H^{2+\varepsilon}\left(\Omega ; \mathbb{R}^{2}\right)
$$

for a fixed $\epsilon>0$ (see [1] for the definition of the Sobolev space $H^{s}\left(\Omega ; \mathbb{R}^{2}\right), s \in \mathbb{R}$ ). The nonsmooth part $\mathbf{u}_{s}$ is of the form

$$
\mathbf{u}_{s}=K_{I} \mathbf{u}_{I}+K_{I I} \mathbf{u}_{I I}
$$

where the vector field $\mathbf{u}_{I}$ (resp. $\mathbf{u}_{I I}$ ) denotes the fracture opening mode (resp. shear mode) and the scalars $K_{I}$ and $K_{I I}$ are the corresponding stress intensity factors. In the following, we assume that

$$
\mathbf{u}_{I}, \mathbf{u}_{I I} \in H^{1+s}\left(\Omega ; \mathbb{R}^{2}\right) \text {, for some } 0<s<1 .
$$

These assumptions are satisfied in the homogeneous case at least when $\boldsymbol{\xi}$ and $\boldsymbol{\zeta}$ are sufficiently smooth, for a straight crack and when the uncracked domain $\Omega^{*}$ has a regular boundary (see [14, 13]). In this case, $s=1 / 2-\eta$ for any $\eta>0$ and the expression of the asymptotic displacement is available in many references such as $[14,17]$. Note that, since $\Omega^{*}$ admits some corners, some 
additional nonsmooth displacements may appear at these corners which may also be taken into account with additional enrichment in an Xfem like approach. We neglect this aspect in this study and consider that the solution is smooth outside the crack tip. Furthermore, we will need the following regularity property

$$
\mathbf{u}_{I}, \mathbf{u}_{I I} \in \mathscr{C}^{\infty}\left(\Omega ; \mathbb{R}^{2}\right)
$$

which is satisfied only for a homogeneous (but not necessarily isotropic) material. When the fracture propagates along the interface between two different materials, the asymptotic displacement $\mathbf{u}_{s}$ can be also written as in (2.5) with more intricate expressions of the two modes $\mathbf{u}_{I}$ and $\mathbf{u}_{I I}$ than in the homogeneous case $[10,28]$.

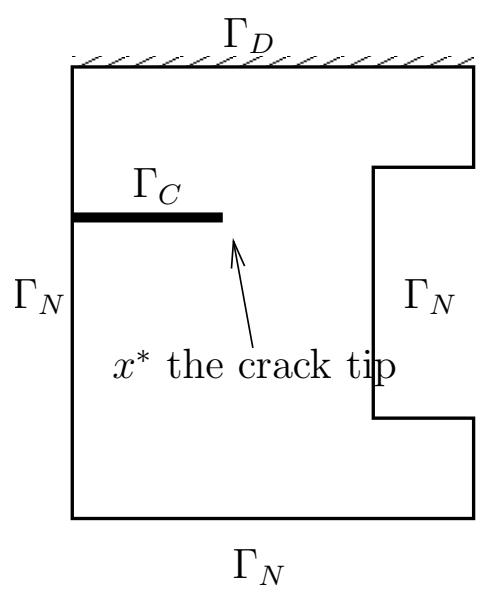

Figure 1: The domain $\Omega$ which represents the reference configuration of a cracked elastic body.

Let $\mathscr{T}_{h}$ be a regular family of triangulations (in the sense of Ciarlet [8]) of the non-cracked domain $\Omega^{*}$ ( $h$ being the mesh parameter). Let us emphasize that the mesh is independent of the crack path. Let $\left(\varphi_{i}\right)_{i \in I}$ be the scalar basis functions of a $P_{1}$ classical finite element method defined on $\mathscr{T}_{h}$. In order to localize the nonsmooth enrichment, we consider $\Omega_{c} \subset \Omega$ a polygonal subdomain of simplified geometry, containing the crack tip $x^{*}$ (see Fig. 2). Let $\chi$ be a cut-off function satisfying

$$
\left\{\begin{array}{l}
\chi(r)=1 \quad \text { if } r<r_{0} \\
0<\chi(r)<1 \quad \text { if } r_{0}<r<r_{1} \\
\chi(r)=0 \text { if } r_{1}<r
\end{array}\right.
$$

where $r_{0}, r_{1}$ are two parameters $\left(0<r_{0}<r_{1}\right)$ such that the ball $B\left(x^{*}, r_{1}\right)$ is included in $\Omega_{c}$. Assume that

$$
\chi \in W^{3, \infty}\left(\Omega^{*}\right)
$$

The definition of the Sobolev space $W^{3, \infty}\left(\Omega^{*}\right)$ can be found in [1]. 
The RB-Xfem displacement field will be defined as

$$
\mathbf{u}^{h}=\sum_{i \in I} \mathbf{a}_{i} \varphi_{i}+\sum_{i \in I_{H}} \mathbf{b}_{i} H \varphi_{i}+\sum_{j=1}^{2} c_{j} \mathbf{w}_{j} \chi
$$

where $\mathbf{a}_{i}, \mathbf{b}_{i} \in \mathbb{R}^{2}$ and $c_{j} \in \mathbb{R}$ are the degrees of freedom. In this expression, the discontinuity of the displacement field along the crack is taken into consideration by the presence of the enrichment functions $\varphi_{i} H$ where $H$ is a step function given by (n denoting a given unit normal to the crack)

$$
H(x)= \begin{cases}+1 & \text { if }\left(x-x^{*}\right) \cdot \mathbf{n} \geq 0, \\ -1 & \text { elsewhere }\end{cases}
$$

and $\left(\varphi_{i}\right)_{i \in I_{H}}$ are the shape functions whose support is totally cut by the crack. Moreover, the vector enrichment functions $\mathbf{w}_{1}$ and $\mathbf{w}_{2}$ are some chosen pre-computed functions defined on $\Omega_{c}$. The motivation of such an enrichment is that the nonsmooth modes $\mathbf{u}_{I}$ and $\mathbf{u}_{I I}$ are often unknown or very complicated (especially in anisotropic or nonhomogeneous cases). Instead, the precomputed functions $\mathbf{w}_{1}$ and $\mathbf{w}_{2}$ will be the result of a finite element computation on $\Omega_{c}$ with a very refined mesh whose parameter is denoted $h^{\prime}$ (see Figure 2). These functions does not have to approximate directly $\mathbf{u}_{I}$ and $\mathbf{u}_{I I}$. The need is that there should exist a linear combination of $\mathbf{w}_{1}$ and $\mathbf{w}_{2}$ whose asymptotic behavior at the crack tip approximate $\mathbf{u}_{I}$ and another one whose asymptotic behavior approximate $\mathbf{u}_{I I}$. Thus, $\mathbf{w}_{1}$ and $\mathbf{w}_{2}$ will be taken to be the solution to two finite element approximations of some elasticity problems posed on $\Omega_{c}$. In other words, $\mathbf{w}_{1}$ and $\mathbf{w}_{2}$ will approximate the solutions $\mathbf{w}_{I}$ and $\mathbf{w}_{I I}$ of two continuous problems. These problems can be chosen to be roughly a opening and a shear experiment on $\Omega_{c}$.

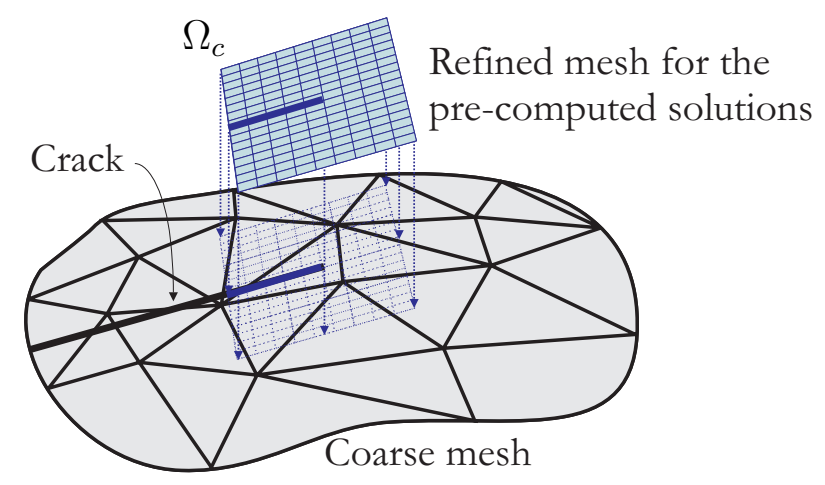

Figure 2: Reduced Xfem enrichment strategy.

The nonsmooth part of the approximated solution (2.10) is

$$
\mathbf{u}_{s}^{h^{\prime}}=\left(c_{1} \mathbf{w}_{1}+c_{2} \mathbf{w}_{2}\right) \chi .
$$


The classical Xfem may become very expensive to model bi-material interface cracks ([28]) because the number of nonsmooth functions generating the exact opening mode and the shear mode increases significantly compared to the homogeneous case ([14], [13]). In RB-Xfem, the number of pre-computed functions will be the same than in the homogeneous case, i.e. only two functions $\mathbf{w}_{1}$ and $\mathbf{w}_{2}$ approximating the exact opening mode and the shear mode. This makes RB-Xfem rather cheaper than the classical Xfem for complex asymptotic displacements.

\section{Error estimates}

The approximate problem can be written

$$
\text { Find } \mathbf{u}^{h} \in \mathbf{V}^{h} \text { such that } a\left(\mathbf{u}^{h}, \mathbf{v}^{h}\right)=l\left(\mathbf{v}^{h}\right) \quad \forall \mathbf{v}^{h} \in \mathbf{V}^{h}
$$

where $\mathbf{V}^{h}$ is the RB-Xfem discrete space given by

$$
\mathbf{V}^{h}=\left\{\mathbf{v}^{h}=\sum_{i \in I} \mathbf{a}_{i} \varphi_{i}+\sum_{i \in I_{H}} \mathbf{b}_{i} H \varphi_{i}+\sum_{j=1}^{2} c_{j} \mathbf{w}_{j} \chi ; \mathbf{a}_{i}, \mathbf{b}_{i} \in \mathbb{R}^{2}, c_{j} \in \mathbb{R}\right\} .
$$

We will denote $\varepsilon_{1}$ (resp. $\varepsilon_{2}$ ) the approximation error between the pre-computed finite element function $\mathbf{w}_{1}\left(\right.$ resp. $\mathbf{w}_{2}$ ) and $\mathbf{w}_{I}$ (resp. $\mathbf{w}_{I I}$ ) such that

$$
\left\{\begin{array}{l}
\mathbf{w}_{1}=\mathbf{w}_{I}+\varepsilon_{1} \\
\mathbf{w}_{2}=\mathbf{w}_{I I}+\varepsilon_{2}
\end{array}\right.
$$

Since $\mathbf{w}_{I}$ (resp. $\mathbf{w}_{I I}$ ) is the exact solution to an elastostatic problem considered on the cracked domain $\Omega_{c}$, it can also be written as follows

$$
\left\{\begin{array}{l}
\mathbf{w}_{I}=\mathbf{w}_{I r}+d_{I}^{1} \mathbf{u}_{I}+d_{I I}^{1} \mathbf{u}_{I I} \\
\mathbf{w}_{I I}=\mathbf{w}_{I I r}+d_{I}^{2} \mathbf{u}_{I}+d_{I I}^{2} \mathbf{u}_{I I}
\end{array}\right.
$$

where $d_{I}^{j}$ and $d_{I I}^{j}$ are some scalars and $\mathbf{w}_{I r}\left(\right.$ resp. $\left.\mathbf{w}_{I I r}\right)$ denotes the regular part of $\mathbf{w}_{I}$ (resp. $\left.\mathbf{w}_{I I}\right)$. We suppose that

$$
\mathbf{w}_{\text {Ir }}, \mathbf{w}_{\text {IIr }} \in H^{2+\epsilon}\left(\Omega_{c} ; \mathbb{R}^{2}\right) .
$$

Let us note that the scalars $d_{I}^{1}$ and $d_{I I}^{1}$ (resp. $d_{I}^{2}$ and $d_{I I}^{2}$ ) are the components of $\mathbf{w}_{I}$ (resp. $\mathbf{w}_{I I}$ ) on the vector spaces vect $\left\{\mathbf{u}_{I}\right\}$ and vect $\left\{\mathbf{u}_{I I}\right\}$. We suppose that

$$
\left|\begin{array}{cc}
d_{I}^{1} & d_{I}^{2} \\
d_{I I}^{1} & d_{I I}^{2}
\end{array}\right| \neq 0
$$

which implies that the two functions $\mathbf{w}_{I}$ and $\mathbf{w}_{I I}$ are linearly independent. 
In order to define a RB-Xfem interpolation operator $\mathscr{I}^{h}$, the nonsmooth part of the interpolate $\mathscr{I}^{h} \mathbf{u} \in \mathbf{V}^{h}$ of the displacement field $\mathbf{u}$ satisfying (2.3), (2.5) can be written

$$
\mathscr{I}_{s}^{h} \mathbf{u}=\sum_{j=1}^{2} c_{j} \mathbf{w}_{j} \chi
$$

where the scalars $c_{j}$ satisfy

$$
\left(\begin{array}{cc}
d_{I}^{1} & d_{I}^{2} \\
d_{I I}^{1} & d_{I I}^{2}
\end{array}\right) \quad\left(\begin{array}{c}
c_{1} \\
c_{2}
\end{array}\right)=\left(\begin{array}{c}
K_{I} \\
K_{I I}
\end{array}\right) .
$$

The regular part of $\mathscr{I}^{h}$ will be defined from the modified regular part of the displacement (2.3)

$$
\mathbf{u}_{r}=\mathbf{u}-\left(c_{1} \mathbf{w}_{I}+c_{2} \mathbf{w}_{I I}\right) \chi .
$$

By using (3.4) and (3.8) we have

$$
\mathbf{u}_{r}=\overline{\mathbf{u}}_{r}+(1-\chi) \mathbf{u}_{s}-\chi\left(c_{1} \mathbf{w}_{\text {Ir }}+c_{2} \mathbf{w}_{\text {IIr }}\right) .
$$

Thanks to the smoothness assumptions (3.5) and (2.9), the functions $\chi \mathbf{w}_{I r}$ and $\chi \mathbf{w}_{I I r}$ satisfy

$$
\chi \mathbf{w}_{\text {Ir }}, \chi \mathbf{w}_{\text {IIr }} \in H^{2+\epsilon}\left(\Omega_{c} ; \mathbb{R}^{2}\right) .
$$

The same holds for $(1-\chi) \mathbf{u}_{s}$ from expression (2.5) and the regularity assumption (2.6). The latter properties, together with condition (2.4) lead to

$$
\mathbf{u}_{r} \in H^{2+\epsilon}\left(\Omega ; \mathbb{R}^{2}\right)
$$

Let $\Omega$ be divided into $\Omega_{1}$ and $\Omega_{2}$ according to the crack and a straight extension of the crack such that the value of $H$ is $(-1)^{k}$ on $\Omega_{k}, k=1,2$. Let $\mathbf{u}_{r}^{k}$ be the restriction of $\mathbf{u}_{r}$ to $\Omega_{k}, k \in\{1,2\}$. As a result of the extension operator theorem (see $[18,1]$ ), there exists an extension $\widetilde{\mathbf{u}}_{r}^{k}$ of $\mathbf{u}_{r}^{k}$ on the uncracked domain $\Omega^{*}$ such that

$$
\widetilde{\mathbf{u}}_{r}^{k} \in H^{2+\epsilon}\left(\Omega^{*} ; \mathbb{R}^{2}\right),\left\|\widetilde{\mathbf{u}}_{r}^{k}\right\|_{2+\epsilon, \Omega^{*}} \leq C_{k}\left\|\mathbf{u}_{r}^{k}\right\|_{2+\epsilon, \Omega_{k}} .
$$

Definition 1. Given a displacement field $\mathbf{u}$ satisfying (2.4), (2.6) and two extensions $\widetilde{\mathbf{u}}_{r}^{1}$ and $\widetilde{\mathbf{u}}_{r}^{2}$ respectively of $\mathbf{u}_{r}^{1}$ and $\mathbf{u}_{r}^{2}$ in $H^{2+\epsilon}\left(\Omega ; \mathbb{R}^{2}\right)$, let $\mathscr{I}^{h} \mathbf{u}$ be the element of $\mathbf{V}^{h}$ such that

$$
\mathscr{I}^{h} \mathbf{u}=\sum_{i \in I} \mathbf{a}_{i} \varphi_{i}+\sum_{i \in I_{H}} \mathbf{b}_{i} H \varphi_{i}+\sum_{j=1}^{2} c_{j} \mathbf{w}_{j} \chi
$$

where $\mathbf{a}_{i}$ and $\mathbf{b}_{i}$ are given as follows ( $x_{i}$ denotes the node associated to $\left.\varphi_{i}\right)$ :

$$
\begin{aligned}
& \text { if } i \in\left\{I \backslash I_{H}\right\} \text {, then } \mathbf{a}_{i}=\mathbf{u}_{r}\left(x_{i}\right), \\
& \text { if } i \in I_{H} \text { and } x_{i} \in \bar{\Omega}_{k} \text { then }(l \neq k)\left\{\begin{array}{l}
\mathbf{a}_{i}=\frac{1}{2}\left(\mathbf{u}_{r}^{k}\left(x_{i}\right)+\widetilde{\mathbf{u}}_{r}^{l}\left(x_{i}\right)\right), \\
\mathbf{b}_{i}=\frac{1}{2}\left(\mathbf{u}_{r}^{k}\left(x_{i}\right)-\widetilde{\mathbf{u}}_{r}^{l}\left(x_{i}\right)\right)(-1)^{k},
\end{array}\right.
\end{aligned}
$$

and $c_{j}$ is defined by (3.8). 
A similar construction of an interpolation operator for Xfem with a cut-off function was introduced in [5]. In the expression (3.14) of the RB-Xfem interpolate, let us denote the regular part by

$$
\mathscr{I}_{r}^{h} \mathbf{u}=\sum_{i \in I} \mathbf{a}_{i} \varphi_{i}+\sum_{i \in I_{H}} \mathbf{b}_{i} H_{i} \varphi_{i}
$$

The values (3.15) of $\mathbf{a}_{i}$ and $\mathbf{b}_{i}$ imply that the regular part $\mathscr{I}_{r}^{h} \mathbf{u}$ coincides with the classical interpolate of $\mathbf{u}_{r}$ (resp. of $\widetilde{\mathbf{u}}_{r}^{k}$ ) on a non-enriched triangle (resp. on a triangle totally enriched by the Heaviside function).

The main modification compared to the definition introduced in [5] is that the nonsmooth part $\sum_{j=1}^{2} c_{j} \mathbf{w}_{j} \chi$ of the RB-Xfem interpolate is an approximation of the exact asymptotic displacement. Moreover, another difference appears in the definition (3.9) of $\mathbf{u}_{r}$ without changing its regularity.

We now give an error estimate essentially based on the result obtained in [5]. In what follows $C$ denotes a generic constant.

Theorem 2. Let $\mathbf{u}$ be the solution to Problem (2.2) satisfying (2.4) and (2.6). Suppose the precomputed functions fulfil the condition (3.5), then

$$
\left\|\mathbf{u}-\mathscr{I}^{h} \mathbf{u}\right\|_{1, \Omega} \leq C\left\{h\left\|\mathbf{u}_{r}\right\|_{2+\epsilon, \Omega}+\left(h^{\prime}\right)^{s}\left(\left\|\mathbf{w}_{I}\right\|_{s+1, \Omega_{c}}+\left\|\mathbf{w}_{I I}\right\|_{s+1, \Omega_{c}}\right)\right\},
$$

where $C:=C(\chi)$ is independent of $h$ and $h^{\prime}$.

Proof. From equations (3.14) and (3.16), one has

$$
\mathscr{I}^{h} \mathbf{u}=\mathscr{I}_{r}^{h} \mathbf{u}+\sum_{j=1}^{2} c_{j} \mathbf{w}_{j} \chi
$$

Then, considering (3.3), one obtains

$$
\mathscr{I}^{h} \mathbf{u}=\mathscr{I}_{r}^{h} \mathbf{u}+\chi\left(c_{1} \mathbf{w}_{I}+c_{2} \mathbf{w}_{I I}\right)+\sum_{j=1}^{2} c_{j} \varepsilon_{j} \chi .
$$

Now, the definition (3.9) implies that the interpolation error is bounded as follows:

$$
\left\|\mathbf{u}-\mathscr{I}^{h} \mathbf{u}\right\|_{1, \Omega} \leq\left\|\mathbf{u}_{r}-\mathscr{I}_{r}^{h} \mathbf{u}\right\|_{1, \Omega}+\sum_{j=1}^{2}\left|c_{j}\right|\left\|\chi \varepsilon_{j}\right\|_{1, \Omega} .
$$

This, together with (3.12) and the convergence result obtained in [5] for the regular part, leads to

$$
\left\|\mathbf{u}-\mathscr{I}^{h} \mathbf{u}\right\|_{1, \Omega} \leq C h\left\|\mathbf{u}_{r}\right\|_{2+\epsilon, \Omega}+\sum_{j=1}^{2}\left|c_{j}\right|\left\|\chi \varepsilon_{j}\right\|_{1, \Omega} .
$$


Now, using the Cauchy-Schwarz inequality and since $\chi$ and its derivatives are bounded, one can write

$$
\left\|\chi \varepsilon_{j}\right\|_{1, \Omega} \leq C\left\|\varepsilon_{j}\right\|_{1, \Omega_{c}} .
$$

Moreover, the pre-computed finite element solutions $\mathbf{w}_{1}$ and $\mathbf{w}_{2}$ approximate displacements $\mathbf{w}_{I}$ and $\mathbf{w}_{I I}$ lying in $H^{s+1}\left(\Omega_{c} ; \mathbb{R}^{2}\right)$ from (3.4), (3.5) and (2.6). Then, the classical convergence results allow to bound the approximation errors $\varepsilon_{1}$ and $\varepsilon_{2}$ as follows (see [8],[11]):

$$
\left\|\chi \varepsilon_{1}\right\|_{1, \Omega} \leq C\left(h^{\prime}\right)^{s}\left\|\mathbf{w}_{I}\right\|_{s+1, \Omega_{c}},
$$

and

$$
\left\|\chi \varepsilon_{2}\right\|_{1, \Omega} \leq C\left(h^{\prime}\right)^{s}\left\|\mathbf{w}_{I I}\right\|_{s+1, \Omega_{c}},
$$

where $h^{\prime}$ is the mesh parameter of the refined mesh used to evaluate the pre-computed functions. Combining the latter estimates with inequality (3.21) allows to conclude.

Remark 3. For a bi-material interface crack, the regularity (2.7) is not satisfied anymore on the interface between the two materials. Therefore, special enrichment functions should be added to capture the displacement field at this interface.

Corollary 4. Let $\mathbf{u}$ be the solution to Problem (2.2) and $\mathbf{u}^{h}$ be the solution to Problem (3.1). With the hypotheses of Theorem 2 and assuming that there exists $c>0$ such that $h^{\prime}<c h^{1 / s}$, we have

$$
\left\|\mathbf{u}-\mathbf{u}^{h}\right\|_{1, \Omega} \leq C h
$$

where $C:=C\left(\mathbf{u}, \chi, \mathbf{w}_{I}, \mathbf{w}_{I I}\right)$ is a constant independent of $h$ and $h^{\prime}$.

The proof is a direct application of the latter theorem together with Cea's lemma.

Remark 5. In the homogeneous case, the asymptotic displacement satisfies $s=1 / 2-\eta$ for any $\eta>0$. In practice, the condition $h^{\prime} \leq c h^{2}$ is sufficient for the optimal convergence.

\section{Numerical experiments}

We perform some numerical experiments in order to compare the RB-Xfem to other Xfem type methods. The numerical computations are achieved using the $\mathrm{C}++$ finite element library Getfem++ [24]. The cracked domain is represented by

$$
\Omega=]-0.5 ; 0.5[\times]-0.5 ; 0.5\left[\backslash \bar{\Gamma}_{C},\right.
$$

where $\Gamma_{C}$ denotes the crack which reads as

$$
\Gamma_{C}=(-0.5 ; 0) \times\{0\}
$$

A $P_{1}$ finite element method is prescribed on a structured mesh of $\Omega$. Figure 3 shows the refined mesh of $\Omega_{c}$ used for the pre-computations. An adapted refinement procedure is performed 
using a classical a posteriori error estimate. This adapted refinement is used in order to reduce the cost of the pre-computations. The pre-computed function $\mathbf{w}_{1}$ and $\mathbf{w}_{2}$ are some finite element approximation of two problems chosen to be approximatively a opening and a shear mode $\Omega_{c}$. For the RB-Xfem computations, the cut-off function used is defined such that

$$
\left\{\begin{array}{l}
\chi(r)=1 \text { if } r<r_{0}=0.01 \\
\chi(r)=0 \text { if } r>r_{1}=0.3
\end{array}\right.
$$

and $\chi$ is identical to a fifth degree polynomial for $r_{0} \leq x \leq r_{1}$.

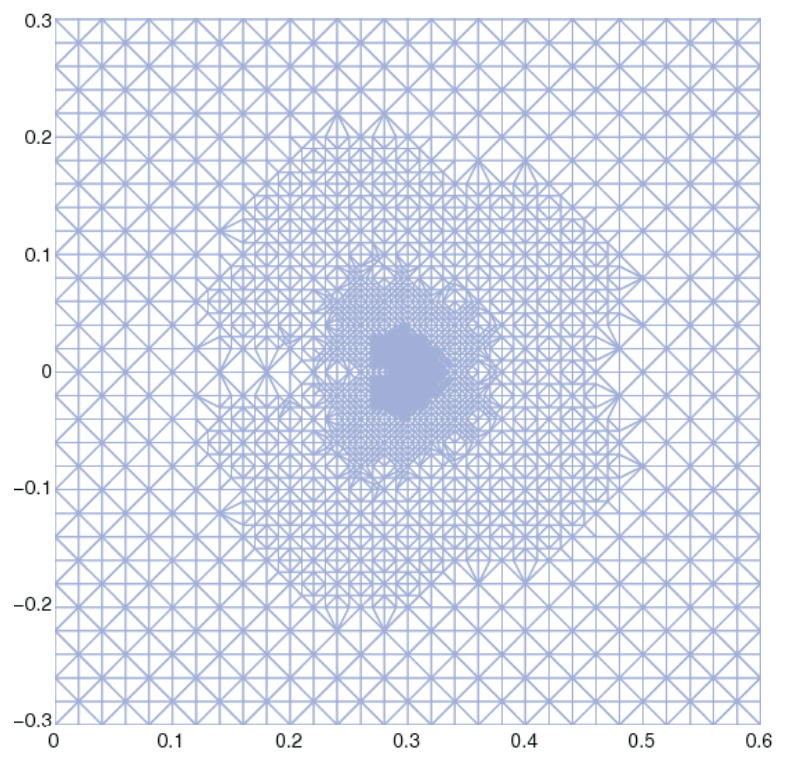

Figure 3: Refined mesh of $\Omega_{c}$ used for the pre-computations.

\subsection{Isotropic homogeneous elastic media}

The first numerical experiment treats an isotropic homogeneous elastic media. Two situations are tested. On the first one, the exact mode I solution (i.e. $u_{I}$ whose expression is well known in this case, see [17] for instance) is prescribed as Dirichlet condition on the boundary of $\Omega$. The second one correspond to a mixed mode problem. The exact solution is a linear combination of the mode I, the mode II and a given regular solution on the non-cracked domain. Figure 7 is a representation of the Von Mises stress on the resulting deformed mesh for the mixed mode problem.

Figures 4 and 5 (respectively Figures 8 and 9) show the convergence curves of the $L^{2}$-norm and the $H^{1}$-norm obtained with the mode I problem (respectively the mixed mode problem) for the classical finite element method, Xfem with surface enrichment [16, 2] and the RB-Xfem. As expected, the error level of Xfem is better than the RB-Xfem since in the latter, the space is enriched 


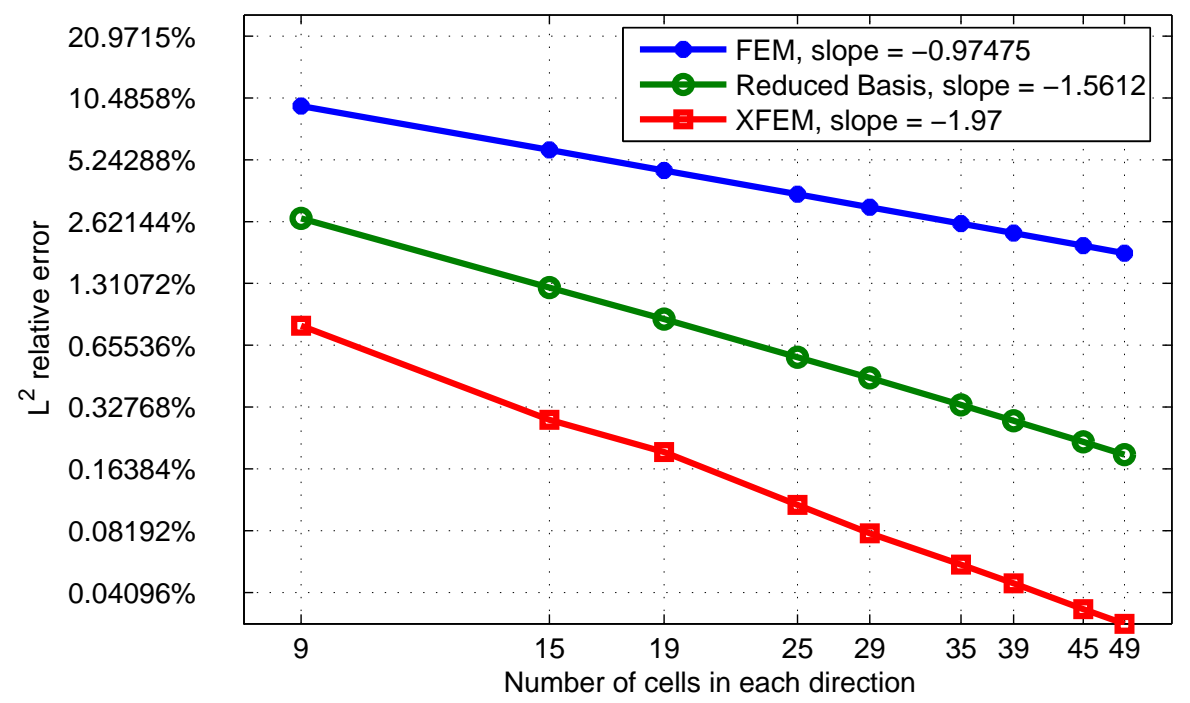

Figure 4: $L^{2}$-error with respect to the number of cells in each direction for a mode I homogeneous crack problem with enriched $P_{1}$ elements (logarithmic scales).

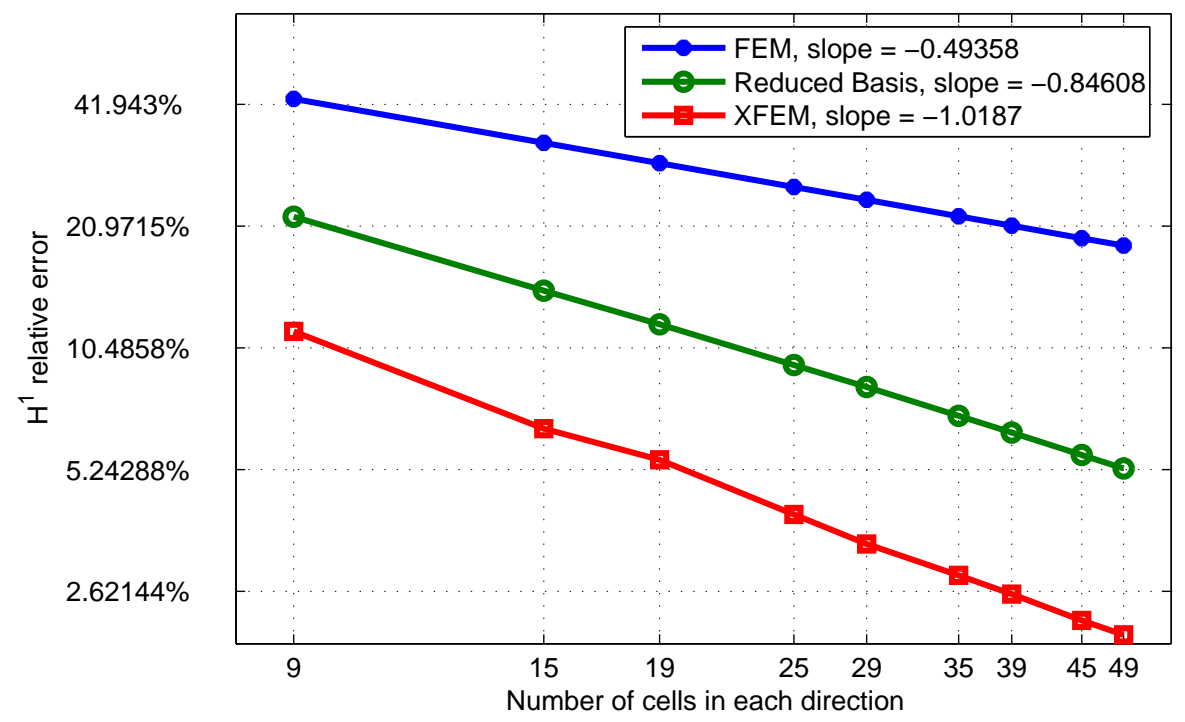

Figure 5: $H^{1}$-error with respect to the number of cells in each direction for a mode I homogeneous crack problem with enriched $P_{1}$ elements (logarithmic scales). 


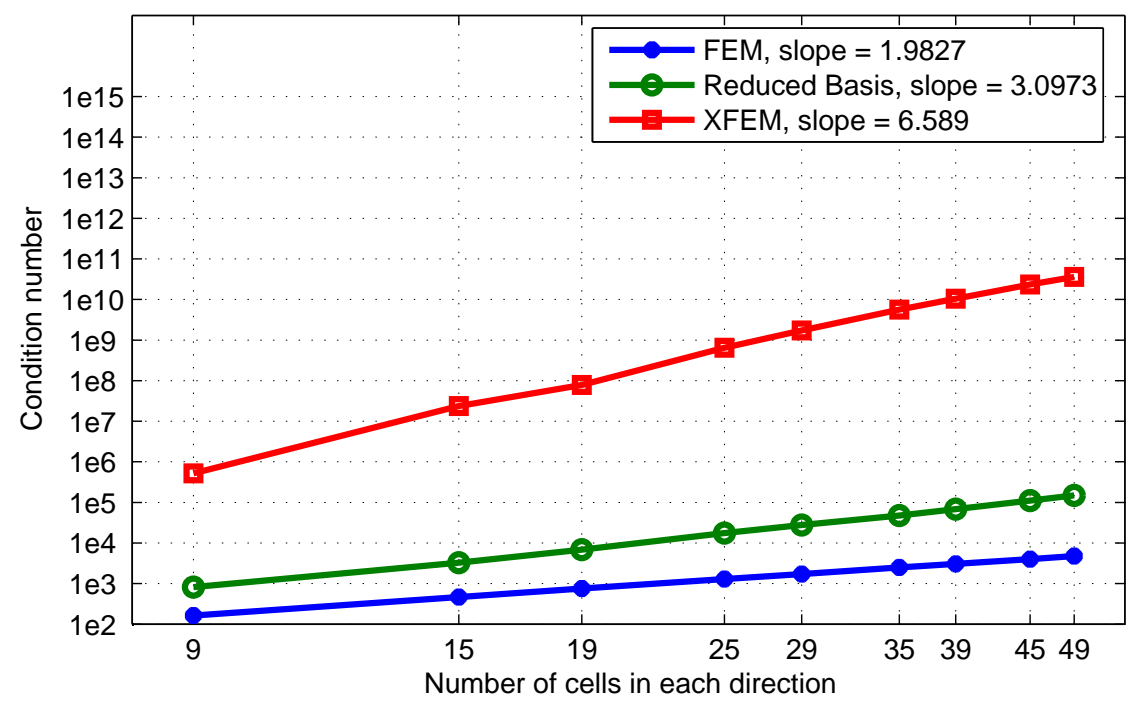

Figure 6: Condition number of the stiffness matrix with respect to the number of cells in each direction for a homogeneous crack.

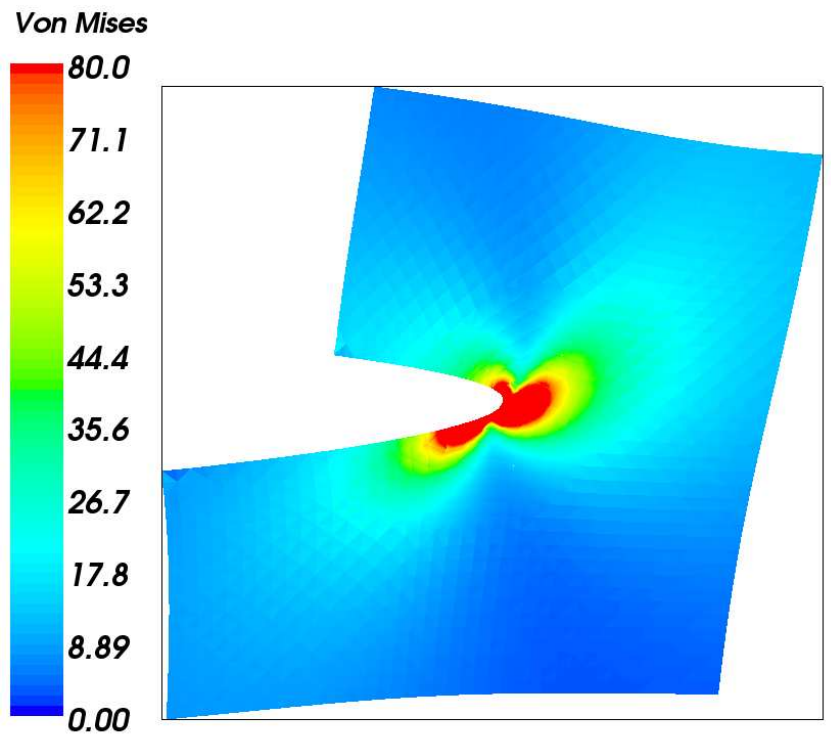

Figure 7: Von Mises stress using $P_{1}$ elements for a mixed mode and an homogeneous crack problem. 


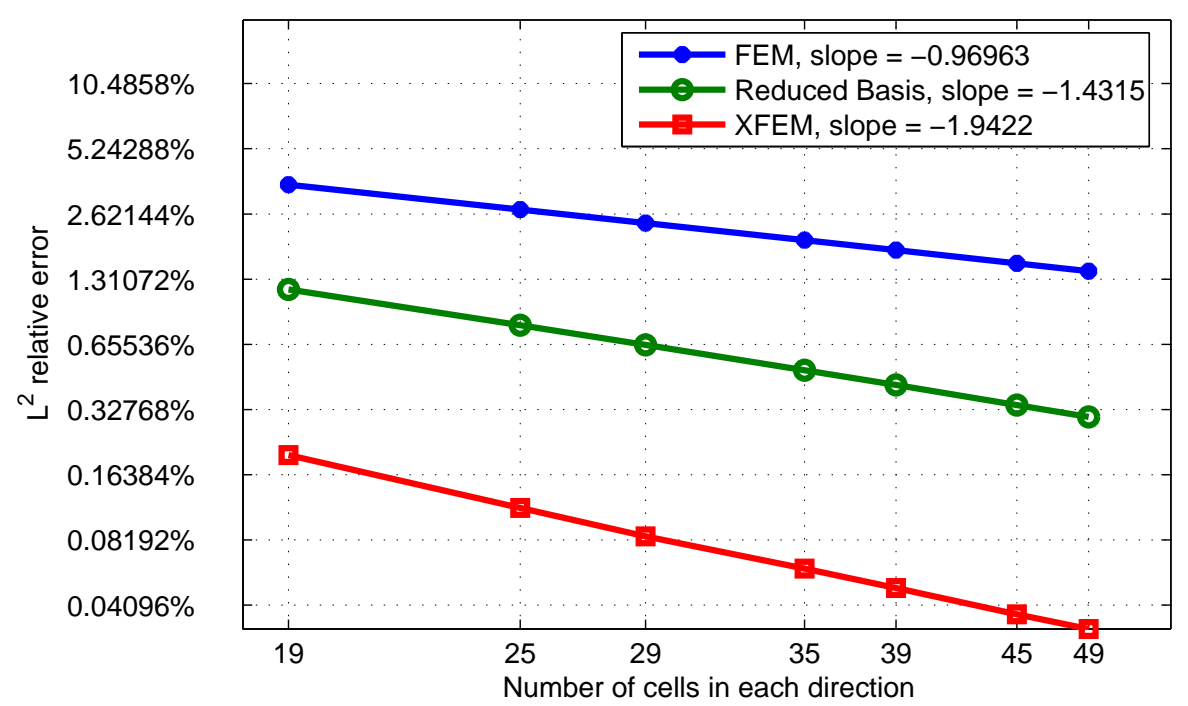

Figure 8: $L^{2}$-error with respect to the number of cells in each direction for a mixed mode homogeneous crack problem with enriched $P_{1}$ elements (logarithmic scales).

by approximations of the asymptotic displacement instead of the exact nonsmooth functions. In the same time, the RB-Xfem reduces significantly the error of the classical finite element method. It shows also a convergence rate very close to the optimal one. Furthermore, the RB-Xfem is introduced via a cut-off function which reduces significantly the number of additional nonsmooth enrichment functions. The condition number of the associated linear system is very much improved compared to Xfem (see Figure 6 for the mode I problem).

\subsection{Bi-material interface crack}

The second numerical experiment is relative to a bi-material interface crack. Non-homogeneous Neumann conditions are prescribed on the boundary. The comparison is done with respect to a refined classical finite element solution. For the sake of simplicity, the discontinuity of the gradient on the interface between the two solids is taken into account by the fact that the interface do not cross the elements. To keep the complete independence of the mesh with respect to the interface it should be necessary to add an enrichment on the part of the mesh where the two material remain stuck by the mean of a function representing the distance to the interface (see [3]).

Figure 10 shows the variation of the Von Mises stress on the deformed structure obtained with RB-Xfem. Figures 11 and 12 show a comparison between the convergence rates of the classical finite element method, the Spider Xfem [6] and the RB-Xfem. The Spider Xfem gives the lower error values since a part of the exact asymptotic displacement is present in the approximation space. Meanwhile, the RB-Xfem error level is only $3 \%$ higher while showing an optimal convergence rate. 


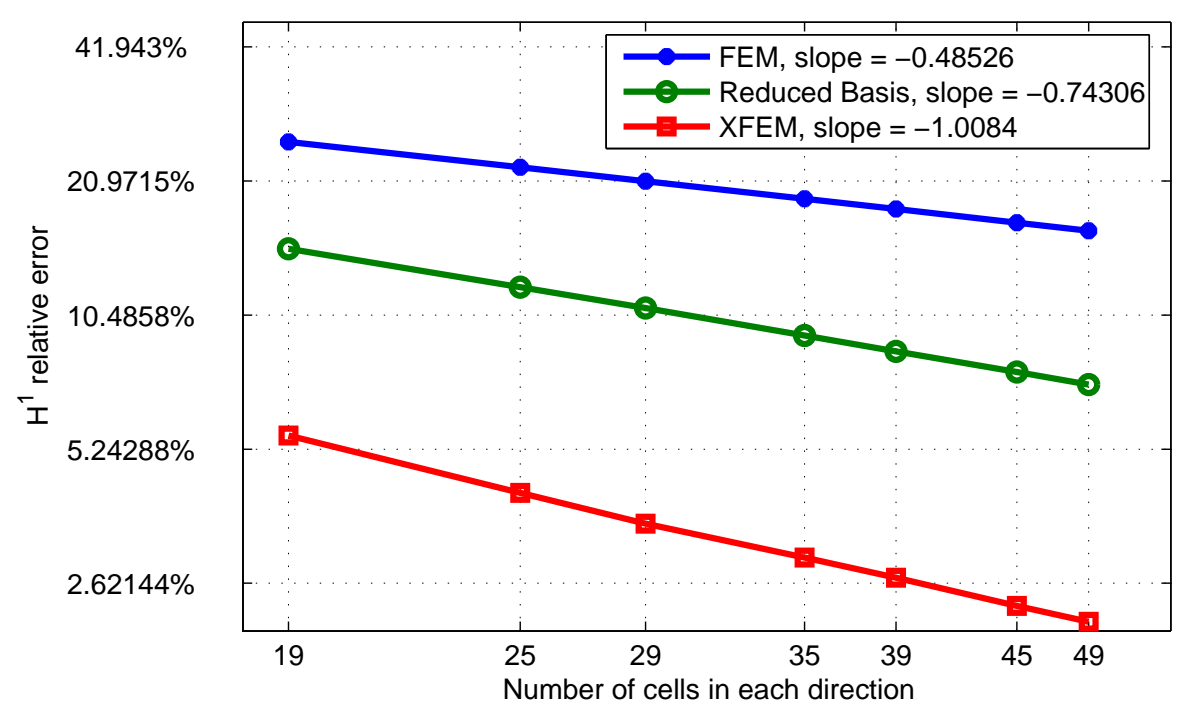

Figure 9: $H^{1}$-error with respect to the number of cells in each direction for a mixed mode homogeneous crack problem with enriched $P_{1}$ elements (logarithmic scales).

The great advantage of RB-Xfem in these experiments is that the optimal performance is obtained without requiring any knowledge on the exact nonsmooth displacement field. Moreover, for complex problems, such as bi-material interface cracks, the RB-Xfem computational cost in terms of number of degrees of freedom is very close to the classical finite element method one since it adds only two vector enrichment functions.

\section{Concluding remarks}

The RB-Xfem offers an interesting framework for modeling complex or even unknown crack asymptotic displacements. The first numerical results are very promising. A better approximation than classical finite element method and an optimal convergence rate are obtained with a reduced computational cost and without any a priori required knowledge on the asymptotic displacement at the crack tip. Therefore, when the asymptotic displacement is unknown, the RB-Xfem is a good alternative since the use of the classical Xfem becomes impossible in such cases. Moreover, the study in the present paper is limited to the RB-Xfem with a cut-off function, but the approach can be extended to the RB-Xfem with integral matching, which is supposed to give better numerical results (see [4] and [7]).

An interesting perspective to this work is the adaptation to large deformations problems and three-dimensional cracks. In this cases, it will be necessary to have a greater number of enrichment functions. 


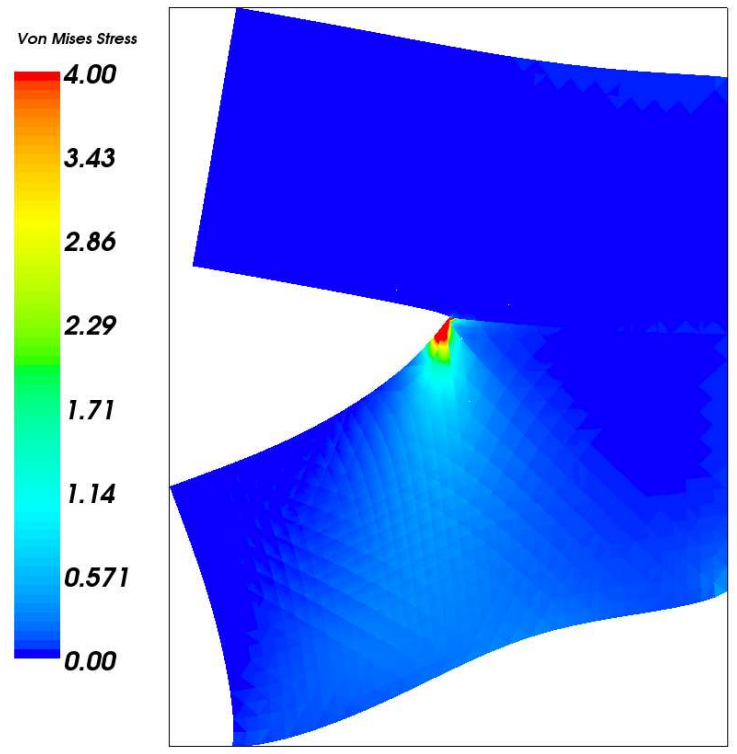

Figure 10: Von Mises stress using $P_{1}$ elements for a bi-material interface crack problem.

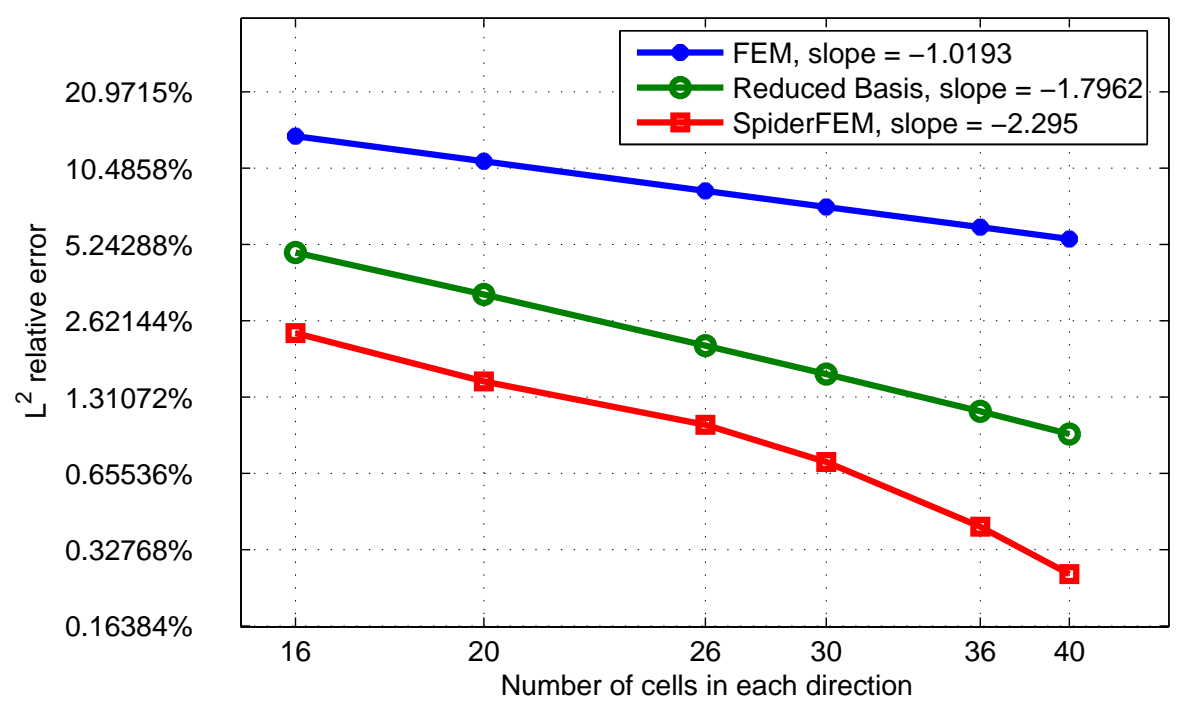

Figure 11: $L^{2}$-error with respect to the number of cells in each direction for a bi-material interface crack problem with enriched $P_{1}$ elements (logarithmic scales). 


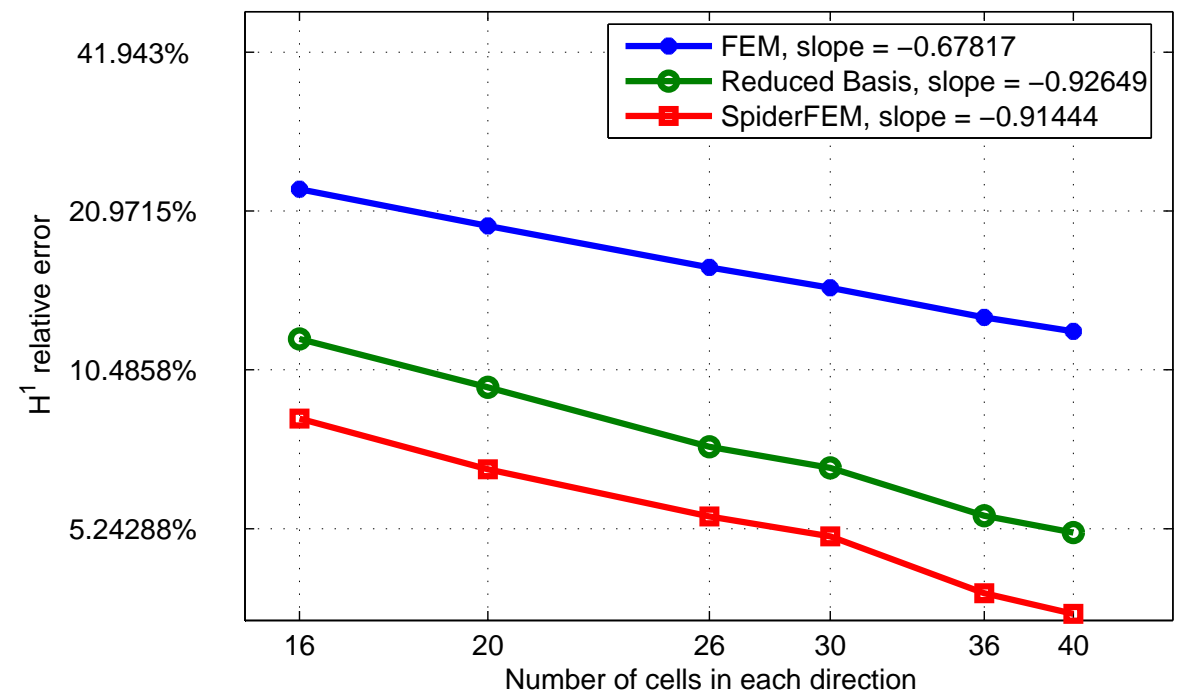

Figure 12: $H^{1}$-error with respect to the number of cells in each direction for a bi-material interface crack problem with enriched $P_{1}$ elements (logarithmic scales).

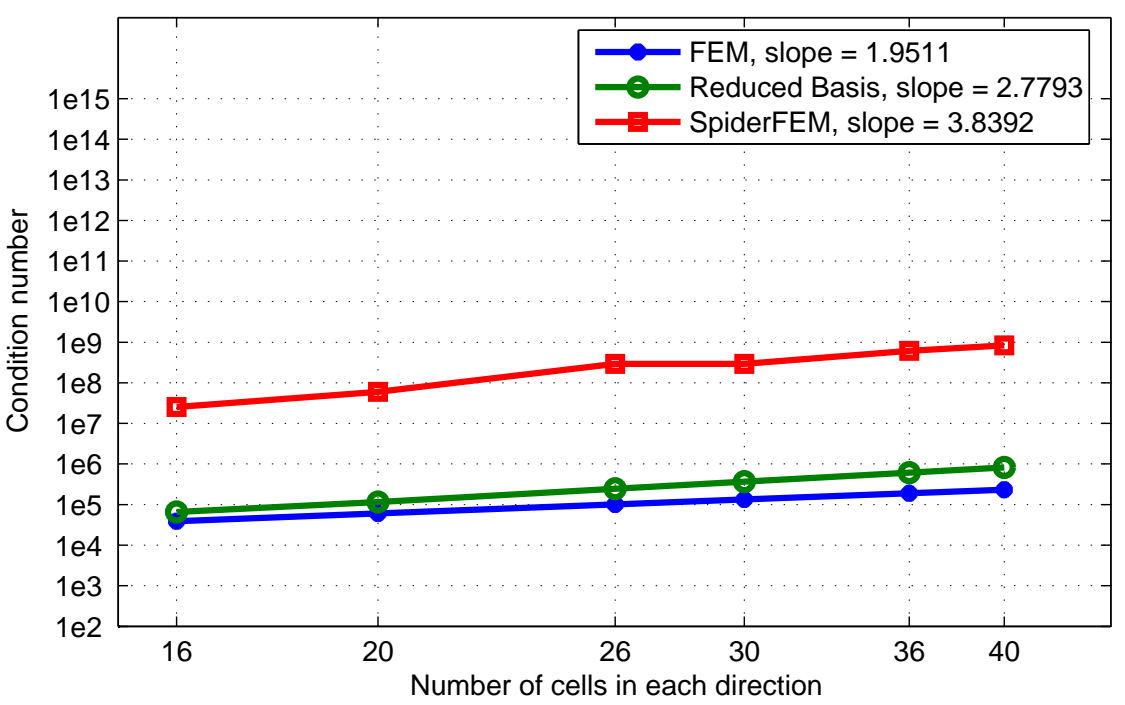

Figure 13: Condition number of the stiffness matrix with respect to the number of cells in each direction for a bi-material interface crack. 
Note that the reduced basis strategy used in the method we propose is limited to the crack tip enrichment, unlike a more conventional use of the reduced basis strategy presented in [15].

This work is supported by "l'Agence Nationale de la Recherche", project ANR-05-JCJC-0182-01.

\section{References}

[1] R.A. Adams. Sobolev Spaces. Academic Press, 1975.

[2] E. Béchet, H. Minnebo, N. Moës, B. Burgardt. Improved implementation and robustness study of the $x$-fem for stress analysis around cracks. Int. J. Numer. Meth. Engng., 64 (2005), 1033-1056.

[3] T. Belytschko, N. Moës, S. Usui, C. Parimi. Arbitrary discontinuities in finite elements. Int. J. Numer. Meth. Engng., 50 (2001), 993-1013.

[4] E. Chahine. Etude mathématique et numérique de méthodes d'éléments finis étendues pour le calcul en domaines fissurés. Thèse de Doctorat de l'INSA de Toulouse, 2008.

[5] E. Chahine, P. Laborde, Y. Renard. Crack-tip enrichment in the Xfem method using a cut-off function. To appear in Int. J. Numer. Meth. Engng.

[6] E. Chahine, P. Laborde, Y. Renard. Spider Xfem: an extended finite element variant for partially unknown crack-tip displacement. To appear in Europ. J. of Comp. Mech.

[7] E. Chahine, P. Laborde, Y. Renard. The extended finite element method with an integral matching condition. Submitted.

[8] P.G. Ciarlet. The finite element method for elliptic problems. Studies in Mathematics and its Applications No 4, North Holland, 1978.

[9] H. Ben Dhia. Multiscale mechanical problems : the Arlequin method. C. R. Acad. Sci., série I, Paris, 326 (1998), 899-904.

[10] M. Dupeux. Mesure des énergies de rupture interfaciale: problématique et exemples de résultats d'essais de gonflement-d'écollement. Mécanique et industrie, 5 (2004), 441-450.

[11] A. Ern, J.-L. Guermond. Éléments finis: théorie, applications, mise en œuvre. Mathématiques et Applications 36, SMAI, Springer-Verlag, 2002.

[12] R. Glowinski, J. He, J. Rappaz, J. Wagner. Approximation of multi-scale elliptic problems using patches of elements. C. R. Math. Acad. Sci., Paris, 337 (2003), 679-684. 
[13] P. Grisvard. Problèmes aux limites dans les polygones - mode d'emploi. EDF Bull. Dirctions Etudes Rech. Sér. C. Math. Inform. 1, MR 87g:35073 (1986), 21-59.

[14] P. Grisvard. Singularities in boundary value problems. Masson, 1992.

[15] D.B.P. Huynh, A.T. Patera. Reduced basis approximation and a posteriori error estimation for stress intensity factors. Int. J. Numer. Meth. Engng. 72 (2007), 1219-1259.

[16] P. Laborde, Y. Renard, J. Pommier, M. Salaün. High order extended finite element method for cracked domains. Int. J. Numer. Meth. Engng., 64 (2005), 354-381.

[17] J. Lemaitre, J.-L. Chaboche. Mechanics of Solid Materials. Cambridge University Press, 1994.

[18] J.L. Lions, E. Magenes. Problèmes aux limites non homogènes et applications, volume 1. Dunod, 1968.

[19] Y. Maday, E.M. Rønquist. A reduced-basis element method. J. Sci. Comput., 17 (2002), (1-4), 447-459.

[20] J.M. Melenk, I. Babuška. The partition of unity finite element method: Basic theory and applications. Comput. Meths. Appl. Mech. Engrg., 139 (1996), 289-314.

[21] N. Moës, T. Belytschko. X-fem: Nouvelles frontières pour les éléments finis. Revue européenne des éléments finis, 11 (1999), 131-150.

[22] N. Moës, J. Dolbow, T. Belytschko. A finite element method for crack growth without remeshing. Int. J. Numer. Meth. Engng., 46 (1999), 131-150.

[23] A.K. Noor, J.M. Peters. Reduced basis technique for nonlinear analysis of structures. AIAA Journal, 18 (2002), No. 4, 455-462.

[24] Y. Renard, J. Pommier. Getfem++. An open source generic C++ library for finite element methods, http://home.gna.org/getfem.

[25] G. Strang, G. Fix. An Analysis of the finite element method. Prentice-Hall, Englewood Cliffs, 1973.

[26] T. Strouboulis, I. Babuska, K. Copps. The design and analysis of the generalized finite element method. Comput. Meths. Appl. Mech. Engrg., 181 (2000), 43-69.

[27] T. Strouboulis, I. Babuska, K. Copps. The generalized finite element method: an example of its implementation and illustration of its performance. Int. J. Numer. Meth. Engng., 47 (2000), 1401-1417.

[28] N. Sukumar, Z. Y. Huang, J.-H. Prévost, Z. Suo. Partition of unity enrichment for bimaterial interface cracks. Int. J. Numer. Meth. Engng., 59 (2004), 1075-1102. 